\title{
Linear drive type of modulated rotating magnetic field for a continuous process of three-dimensional crystal orientation
}

\author{
Shigeru HORII ${ }^{1, \dagger}$, Itsuki ARIMOTO ${ }^{1}$ and Toshiya DOI ${ }^{1}$ \\ ${ }^{1}$ Graduate School of Energy Science, Kyoto University, Yoshida-Honmachi, Sakyo-ku, Kyoto 605-8501, Japan
}

Equipment that can generate a linear drive type of modulated rotating magnetic field (MRF) was developed for three-dimensional magnetic alignment applicable to a continuous production process. This equipment consists of an arrayed magnet unit assembled using permanent magnets and a linear drive system, and it can generate a static magnetic field region with $0.9 \mathrm{~T}$ and a rotating magnetic field region with $0.5-0.9 \mathrm{~T}$ and $520 \mathrm{rpm}$. The equipment has been used to successfully biaxially align powders of an orthorhombic cuprate superconductor $\mathrm{DyBa}_{2} \mathrm{Cu}_{3} \mathrm{O}_{y}(y \sim 7)$ with twin microstructures in epoxy resin at room temperature without rotating the sample. (O2018 The Ceramic Society of Japan. All rights reserved.

Key-words: Magnetic alignment, Linear drive, Modulated rotating magnetic field, $\mathrm{REBa}_{2} \mathrm{Cu}_{3} \mathrm{O}_{y}$, Superconductor

[Received June 25, 2018; Accepted September 13, 2018; Published online October 4, 2018]

A crystal orientation technique using magnetic field enables alignment of the magnetization axis of grain in a microstructure to a designed direction; therefore, it is important for ceramics with anisotropic functionalities. The formation of a triaxial oriented microstructure is expected to lead to excellent properties almost equal to those of the corresponding single crystal in the resultant ceramics. Triaxial crystal orientation is achievable by using a modulated rotating magnetic field $(\mathrm{MRF})^{1)-6)}$ in a roomtemperature process. This leads directly to a production process for fabricating a pseudo-single-crystal material as well as an epitaxial growth technique. Triaxial crystal orientation using MRF is accomplished by the magnetic alignment of two magnetization axes: the first easy axis of magnetization by a static magnetic field and the hard axis of magnetization by a rotating magnetic field. Various types of MRFs, such as the modulated rotation speed type, ${ }^{2), 3)}$ the intermittent rotation type, ${ }^{4), 5)}$ and the oscillating rotation type, $\left.{ }^{6}\right)$ have been reported. Substances with a triaxial magnetic anisotropy under the MRF that have sufficiently high orientation energy are biaxially aligned. In practice, biaxial magnetic alignment has been demonstrated in substances with an orthorhombic crystal structure such as $\mathrm{RE}_{2} \mathrm{Ba}_{4} \mathrm{Cu}_{7} \mathrm{O}_{y}$ (RE: rare-earth element), 1),3),4),7) $\mathrm{REBa}_{2} \mathrm{Cu}_{4} \mathrm{O}_{8}{ }^{4)-6), 8)}$ and L-alanine. ${ }^{2)}$ Figure 1(a) shows a schematic of an intermittent type of MRF as an example. This includes a resting process every 180 degrees in a rotating magnetic field. ${ }^{4), 7), 8)}$ When the sample's original angle, $0^{\circ}$, was defined with regard to a direction normal to the $\alpha$ plane [see Fig. 1(a)] of the sample, the first easy, second easy, and hard axes of magnetization are oriented

\footnotetext{
Corresponding author: S. Horii; E-mail: horii.shigeru.7e@ kyoto-u.ac.jp
}

normal to the $\alpha, \beta$, and $\gamma$ planes, respectively, in principle.

To generate an MRF, a process of rotating a magnetic field or that of a sample in a static magnetic field is required. Therefore, it is not easy to introduce rotating magnetic fields into a continuous production process with linear motion. The development of a device that generates an MRF without rotating the specimen or the magnetic field is important for enhancing the versatility of the magnetic alignment technique as a material production process. Particularly, it is useful for a continuous production process with a linear uniaxial motion. As reported in this paper, we developed an arrayed permanent magnet unit that can generate an MRF by the linear motion of the unit alone. In addition, as a proof of principle, the powder of an REbased high-critical-temperature superconductor (HTSC), ${ }^{9)}$ $\mathrm{DyBa}_{2} \mathrm{Cu}_{3} \mathrm{O}_{y}$, with an orthorhombic crystal structure and twin microstructure in its grains was aligned at room temperature in the epoxy resin. The requirements for practical use of the HTSC are the formation of a biaxial orientation ${ }^{10)}$ and a large cross-sectional area, leading to high critical currents. To realize a long HTSC wire by magnetic alignment, the development of a device that can generate a linear drive type of MRF is important. It is expected that by combining the use of this device with the colloidal process, a thick-filmed HTSC wire with a high degree of biaxial orientation can be produced. Furthermore, it is expected that the device can be applied not only to achieving hightemperature superconductivity but also to orienting the filler in resin and in an anisotropic functional material processed by sheet casting.

Figure 1(b) shows a schematic of the above equipment. Its outside dimensions are $550 \mathrm{~mm} \mathrm{~W} \times 368 \mathrm{~mm} \mathrm{D} \times$ $216 \mathrm{~mm} \mathrm{H}$. The rotational motion of a rotor is converted into the linear motion of the arrayed permanent magnet unit 
(a)

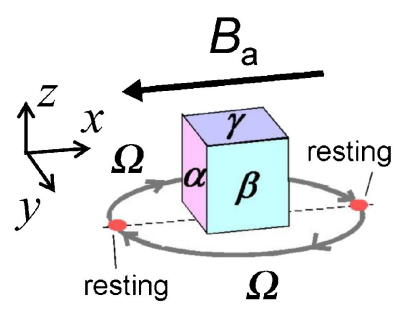

(b)

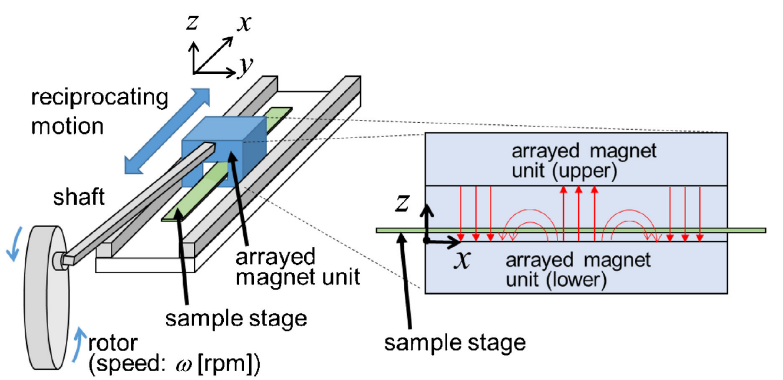

Fig. 1. Schematics of (a) the intermittent type of MRF and (b) the equipment of the linear drive type of MRF. $B_{\mathrm{a}}$ and $\Omega$ in Fig. 1(a) are the external magnetic flux density and the rotation speed of $B_{\mathrm{a}}$, respectively. Three planes of a magnetically oriented powder sample, the $\alpha, \beta$ and $\gamma$ planes, are used for evaluation of the orientation degrees by X-ray diffraction.

(a)
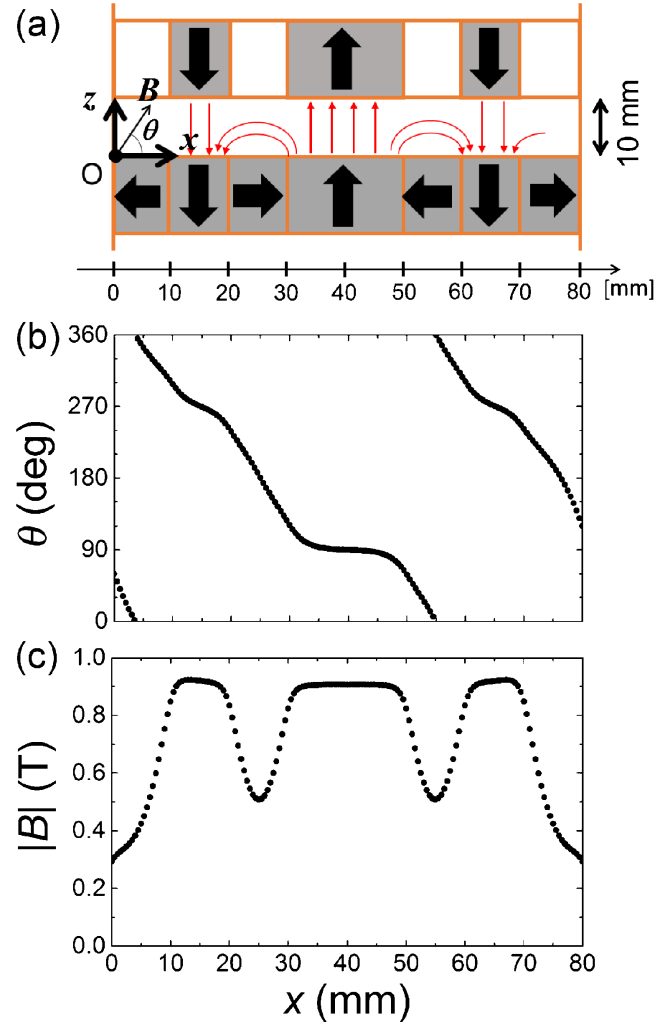

Fig. 2. (a) Schematic of the cross section of the arrayed magnet unit; changes in (b) $\theta$ and (c) $|B|$ at $z=2 \mathrm{~mm}$ for the arrayed magnet unit as a function of $x$. The black arrows in boxes and red arrows in Fig. 2(a) show the magnetized directions of permanent magnets in the arrayed magnet unit and the magnetic flux generated between the upper and lower parts of the arrayed magnet unit.

$80 \mathrm{~mm} \mathrm{~W} \times 36 \mathrm{~mm} \mathrm{D} \times 64 \mathrm{~mm} \mathrm{H}$. The length of its linear reciprocating motion can be controlled by the position in which a shaft is mounted to the rotor. Schematics of the cross section of the arranged magnet unit and the expected magnetic flux in a gap between the upper and lower parts of the arranged magnetic unit are also shown in Fig. 1(b). Qualitatively, the arranged magnet unit requirement for the generation of an MRF is the co-existence of a spatially homogeneous magnetic flux and a bent magnetic flux in a gap between the upper and lower parts of the unit. Both static and rotating fields can be generated in the gap by the reciprocated motion of the unit. Figures 2(a)-2(c) show a schematic of the cross section of the arrayed magnet unit, the change in the angle of the magnetic field strength, and the distribution of the absolute magnetic field strength as a function of the horizontal position, respectively. The arrows in Fig. 2(a) represent the directions of magnetization for permanent $\mathrm{Nd}-\mathrm{Fe}-\mathrm{B}$ magnets in the arrayed magnet unit. The results of Figs. 2(b) and 2(c) were obtained using a calculation in the finite element method. Note that the horizontal position, $x$, and vertical position, $z$, in Figs. 2(b) and 2(c) are defined as distances in the horizontal and vertical directions from the left end of the top surface of the lower magnets [see Fig. 2(a)], respectively, and the magnetic field direction, $\theta$, is defined as the angle tilted in a counterclockwise direction from the $x$ direction. The directions of $x$ and $z$ correspond with those in Fig. 1(b). In the present study, the bottoms of samples were mounted at a position where $x=40 \mathrm{~mm}$ and $z=2 \mathrm{~mm}$, and the linear reciprocating motion of the arrayed magnet unit was repeated in the region of $15[\mathrm{~mm}]<x<65[\mathrm{~mm}]$.

As shown in Fig. 2(a), $\theta$ at the space between the upper and lower parts of the arrayed magnet unit was controlled by arranging the magnets so that they are magnetized in different directions. A pair of magnets arranged in the $z$-axis direction with two permanent magnets magnetized vertically upward is located at $x=40 \mathrm{~mm}$. Two pairs of magnets magnetized vertically downward are located at $x=15$ and $65 \mathrm{~mm}$. Horizontally magnetized magnets were placed at intervals at the lower side of the magnet array. Consequently, part of the magnetic flux in the pair of magnets at $x=40 \mathrm{~mm}$ is bent toward the adjacent pairs of magnets as schematically illustrated with red arrows in Fig. 2(a). Therefore, there are two different regions in this arrayed magnet unit. One is a magnetic field that exists in the vertical direction with almost homogeneous magnetic flux density. The other is a magnetic field bent toward the adjacent opposing magnet. By linearly reciprocating the motion of the arrayed magnet unit in the $x$ direction, static and rotating magnetic fields are expected to be generated in the former and latter regions. As can be seen in Fig. 2(b), the static magnetic field region is $35[\mathrm{~mm}]<$ $x<45[\mathrm{~mm}]$, and the rotating magnetic field regions are $15[\mathrm{~mm}]<x<35[\mathrm{~mm}]$ and $45[\mathrm{~mm}]<x<65[\mathrm{~mm}]$.

Figure 2(c) shows the $x$ dependence of the absolute value of the magnetic field strength $(|B|)$ at $z=2 \mathrm{~mm}$ for 
the arrayed magnet unit. $|B|$ in the static field region is approximately $0.9 \mathrm{~T}$. On the other hand, $|B|$ in the rotating field region varies with $x$, and the minimum $|B|$ was approximately $0.5 \mathrm{~T}$. Therefore, in the present equipment, the orientation energy generated in the rotating field region is relatively small. However, the $|B|$ of the static and rotating field regions can be controlled by optimizing the spacing between the upper and lower parts of the arrayed magnet unit, the widths of the upper part of the arrayed magnet unit, and the magnets magnetized in the horizontal direction. This optimization leads to an arrayed magnet unit appropriate for each triaxial magnetic anisotropy to maximize the triaxial orientation degrees.

On the other hand, one feature of the present equipment is that an effective rotation speed generated in the rotating magnetic field region $(\Omega)$ is high. The maximum value of $\Omega$ was equivalent to approximately $520 \mathrm{rpm}$ for the rotational speed of the rotor at $180 \mathrm{rpm}$. In the case of the sample-rotation type of intermittent MRF used in our group, ${ }^{11)}$ the maximum value of the sample-rotation speed was $60 \mathrm{rpm}$. That is, the MRF in the present equipment can be applied at an $\Omega$ nine times higher than that without rotating the sample. This is advantageous for aligning substances with high magnetic anisotropies by using the rotating field. There are two types of particle orientations in a rotating magnetic field. ${ }^{12}$ ) One is such that the first easy magnetization axis can follow the rotating magnetic field. The other is the hard magnetization axis alignment mentioned above. Qualitatively, with an increase in the rotating speed, the alignment mode is shifted from the former mode to the latter mode. One can recognize that the present equipment can be applied to a linear-movement production process and can apply an MRF with a higher $\Omega$. Incidentally, in principle, the first easy axis of magnetization is aligned in the $z$-axis direction in Fig. 2(b), and the hard axis of magnetization is aligned in a direction perpendicular to the $x$ - and $z$-axes.

Polycrystals of $\mathrm{DyBa}_{2} \mathrm{Cu}_{3} \mathrm{O}_{y}$ (Dy123, $y \sim 7$ ) with the orthorhombic crystal structure were synthesized via a standard solid-state reaction in air. The starting materials$\mathrm{Dy}_{2} \mathrm{O}_{3}, \mathrm{BaCO}_{3}$, and $\mathrm{CuO}$-were weighed at a molar ratio of 1:2:3, respectively, and ground thoroughly in ethanol. The mixed powders were heat-treated twice at 860 and $880^{\circ} \mathrm{C}$ with intermediate grindings. They were then pelletized and sintered at $960^{\circ} \mathrm{C}$ for $24 \mathrm{~h}$ to promote grain growth during the sintering process. The obtained Dy123 pellets were crushed in an agate mortar to fabricate powders for magnetic alignment. A scanning electron microscope (SEM) was used to determine the volume-weighted mean diameters $(d)$ of the Dy123 powders. The determined $d$ value for the Dy123 powder was $13.1 \mu \mathrm{m}$. Incidentally, a fractured surface was also observed by SEM to confirm the grain size of the Dy 123 pellet after sintering at $960^{\circ} \mathrm{C}$ in air. The maximum grain size of the sintered Dy123 pellet was $>30 \mu \mathrm{m}$. This result indicates that sufficient grain growth was achieved by the sintering at $960^{\circ} \mathrm{C}$, and most of the crushed Dy 123 particles consisted of a single grain.

Using the above equipment, the Dy123 powder was aligned in epoxy resin at room temperature. The rotation speeds of the crank rotor $(\omega)$ were $180 \mathrm{rpm}$ (the maximum rotation speed) and $10 \mathrm{rpm}$, and the bottom parts of the samples were mounted at $z=2 \mathrm{~mm}$. The samples were $10 \mathrm{~mm} \mathrm{~W} \times 10 \mathrm{~mm} \mathrm{D} \times 1.5 \mathrm{~mm} \mathrm{H}$. The effective application times of the static field $\left(t_{1}\right)$ and rotating field $\left(t_{2}\right)$ per half of one reciprocation for $\omega=180$ and $10 \mathrm{rpm}$ were $\left(t_{1}, t_{2}\right)=(0.05 \mathrm{~s}, 0.12 \mathrm{~s})$ and $(0.93 \mathrm{~s}, 2.07 \mathrm{~s})$, respectively. Here, as can be seen in Fig. 2(b), half of one reciprocation in the region of $15[\mathrm{~mm}]<x<65[\mathrm{~mm}]$ is equivalent to one rotation of the magnetic flux. Therefore, for comparison with the latter case, a Dy123 power sample $13 \mathrm{~mm}$ $\mathrm{W} \times 13 \mathrm{~mm} \mathrm{D} \times 13 \mathrm{~mm} \mathrm{H}$ was also fabricated under an intermittent type of MRF with $1 \mathrm{~T}, t_{1}=1 \mathrm{~s}$, and $t_{2}=2 \mathrm{~s}$ $(\Omega=30 \mathrm{rpm})$ in a rotation of the sample using a superconducting solenoidal magnet. ${ }^{11)}$ In both experimental studies, Araldite ${ }^{\circledR}$ Standard (Huntsman, Japan) was used as an epoxy resin and its curing time was $12 \mathrm{~h}$. The volume fractions of all Dy123 powder samples were 5 vol.\% and their samples were obtained without sedimentation of the Dy123 powders.

To determine the degrees of the $c$-axis and in-plane orientation for completely cured resins containing magnetically aligned powders (magnetically aligned powder samples) of Dy123, pole figures of the (103) plane were examined. The biaxial orientation degree, $F$, was determined from the results of the (103) pole figures. In detail, the sums of intensities of the four 103 peaks $\left(\Sigma I_{103}\right)$ and for a whole measured region $\left(\Sigma I_{\text {all }}\right)$ were determined, and $F=\Sigma I_{103} / \Sigma I_{\text {all }}$ was calculated as the index of the biaxial orientation degree. Note that the sum for the 103 peaks was examined in the measured region within 5 degrees of the radius of each 103 peak.

Figure 3 shows the (103) pole figure of the Dy123 powder sample oriented at $\omega=180 \mathrm{rpm}$ for the rotor using the above equipment. The measurement was performed for a plane normal to the $z$-axis in Fig. 2(b). Four-fold symmetric peaks were observed. This result was qualitatively in agreement with the result as measured at the $\alpha$ plane [see Fig. 1(a)] for the Dy123 powder sample aligned with $10 \mathrm{~T}$ of an intermittent type of MRF using a superconducting solenoidal magnet. ${ }^{11)}$ In the case of the biaxial alignment of a substance with an orthorhombic crystal structure, two-fold symmetric peaks appear. However, a twin microstructure is introduced in the RE123 particles processed by the oxygen annealing, ${ }^{13}$ ) and the result in Fig. 3 can be understood as the biaxial orientation of Dy123 particles having a twin microstructure. Therefore, it was demonstrated that the linear reciprocation of the

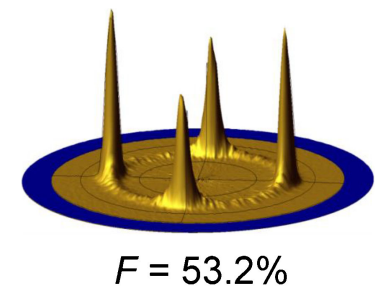

Fig. 3. A (103) pole figure of the Dy123 powder sample oriented under the linear drive type of MRF with $\Omega=520 \mathrm{rpm}$. 


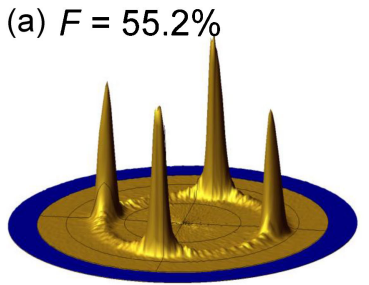

(b) $F=76.7 \%$

Fig. 4. The (103) pole figures of Dy123 powder samples aligned under (a) the linear drive type of MRF with $\left(t_{1}, t_{2}\right)=$ $(0.93 \mathrm{~s}, 2.07 \mathrm{~s})$ and $(\mathrm{b})$ the intermittent type of MRF of $1 \mathrm{~T}$ with $\left(t_{1}, t_{2}\right)=(1 \mathrm{~s}, 2 \mathrm{~s})$.

arrayed magnet unit could properly generate an MRF. Incidentally, the value of the orientation degree, $F$, was $53 \%$. In detail, it was found from the (103) pole figure in Fig. 3 that the alignment of twinned Dy123 powders with $c / / z, a(b) / / x$ and $b(a) / / y$ [see Fig. 1(b)] was accomplished. From the (103) pole figure in Ref. 11, the alignment with $c / / x \perp \alpha, a(b) / / y \perp \beta$ and $b(a) / / z \perp \gamma$ [see Fig. 1(a)] was determined. However, the $c$ - and $b$-axes of the Dy123 powder were aligned as the effects of static and rotating magnetic fields, respectively, in both cases.

In order to understand the orientation degrees of Dy 123 powder samples aligned under the linear drive type of MRF, the powder samples were fabricated in almost the same condition of $t_{1}$ and $t_{2}$ under the linear drive type of MRF and the intermittent type of MRF using the superconducting solenoidal magnet, and these $F$ values were compared. Figures 4(a) and 4(b) show the (103) pole figures of the Dy123 powder samples aligned under the linear drive type of MRF with $\left(t_{1}, t_{2}\right)=(0.93 \mathrm{~s}, 2.07 \mathrm{~s})$ and under the intermittent type of MRF of $1 \mathrm{~T}$ with $\left(t_{1}, t_{2}\right)=$ $(1 \mathrm{~s}, 2 \mathrm{~s})$, respectively. Note that the measurement was performed at the $c$-axis-oriented surface. In both cases, four-fold symmetric peaks appeared, and the Dy123 powders were biaxially oriented. However, the $F$ values were 55.2 and $75.2 \%$ for Figs. 4(a) and 4(b), respectively. The degree of orientation in the Dy123 powder sample aligned using the superconducting magnet was higher than that using the linear drive type of MRF. As described in Fig. 2, the linear drive type of MRF generates $0.9 \mathrm{~T}$ in the static field region; however, a clearly lower magnetic field, $0.5 \mathrm{~T}$, is generated in the rotating field region. That is, the $|B|$ of the rotating magnetic field region is approximately half that of the superconducting magnet. It is likely that the lowering of the rotating field region led to a decrease in the orientation degree in the case of the linear drive type of MRF. It was reported in our previous work that high degrees of $c$-axis orientation for Dy123 were maintained down to $0.5 \mathrm{~T}$; however, the in-plane orientation degrees were drastically lowered below $1 \mathrm{~T}$. ${ }^{11)}$ When focusing closely on the result in Fig. 4(a), the four peaks of the (103) pole figure were broadened only in the direction of the rotational angle. This broadening means a decrease in the in-plane orientation degree. Therefore, the results in Fig. 4(a) are qualitatively consistent with our speculation.

In conclusion, we developed a device that can generate a linear drive type of MRF for a continuous production process. In the case of the arrayed magnet unit assembled using permanent magnets in the present study, the $|B|$ values in the static and rotating field regions were approximately $0.9 \mathrm{~T}$ and $0.5-0.9 \mathrm{~T}$, respectively. Furthermore, the $\Omega$ value achieved approximately $520 \mathrm{rpm}$ at the maximum. It was found that the Dy123 powder was biaxially oriented in the epoxy resin at room temperature using this equipment. At the current stage, the improvement of $|B|$ in the rotating magnetic field region, such as optimizing the arrangement of the arrayed magnet unit and utilizing the superconducting bulk magnet, is required. If this issue is overcome, the applicable substances will be increased drastically, and versatility will be further enhanced. Furthermore, in order to form biaxially oriented Dy123 tape using a Dy123 slurry and this apparatus, control of the viscosity $(\eta)$ and time-dependence of $\eta$ for the slurry is important. This is because the first easy magnetization axis follows the rotating magnetic field more easily in the case of a dispersant with lower $\eta$, and this does not lead to the alignment of the hard magnetization axis by the rotating magnetic field. It is expected that the orientation degrees of biaxially oriented Dy123 tape will be improved by understanding the time-dependence of $\eta$ for the Dy123 slurry and optimization of the arrayed magnet unit.

Acknowledgement This work was partly supported by the Adaptable and Seamless Technology Transfer Program through Target-Driven R\&D (A-STEP), the Japan Science and Technology Agency (JST), and JSPS KAKENHI Grant Number JP17H03235. The authors thank Mr. Suzuki and Mr. Sugaya, TOWA Co., Ltd., Japan, for their kind support in calculations using the finite element method.

\section{References}

1) S. Stains, J. Genoud, A. Mawdsley and V. Manojlovic, IEEE Trans. Appl. Supercond., 9, 2284-2287 (1999).

2) T. Kimura and M. Yoshino, Langmuir, 21, 4805-4808 (2005).

3) T. Fukushima, S. Horii, H. Ogino, T. Uchikoshi, T. S. Suzuki, Y. Sakka, J. Shimoyama and K. Kishio, Appl. Phys. Express, 1, 111701 (2008).

4) S. Horii, M. Yamaki, H. Ogino, T. Maeda and J. Shimoyama, Physica C, 470, 1056-1059 (2010).

5) M. Yamaki, S. Horii, M. Haruta and J. Shimoyama, Jpn. J. Appl. Phys., 51, 010107 (2012).

6) M. Yamaki, M. Furuta, T. Doi, J. Shimoyama and S. Horii, Phys. Procedia, 58, 62-65 (2014).

7) S. Horii, S. Okuhira, M. Yamaki, K. Kishio and J. Shimoyama, J. Appl. Phys., 115, 113908 (2014).

8) S. Horii, M. Yamaki, J. Shimoyama, K. Kishio and T. Doi, Supercond. Sci. Tech., 28, 105003 (2015).

9) M. K. Wu, J. R. Ashburn, C. J. Torng, P. H. Hor, R. L. Meng, L. Gao, Z. J. Huang, Y. Q. Wang and C. W. Chu, Phys. Rev. Lett., 58, 908-910 (1987).

10) D. Dimos, P. Chaudhari and J. Manhart, Phys. Rev. B, 41, 4038-4049 (1990).

11) S. Horii, T. Nishioka, I. Arimoto, S. Fujioka and T. Doi, Supercond. Sci. Tech., 29, 125007 (2016).

12) T. Kimura, Polym. J., 35, 823-843 (2003).

13) Z. Hiroi, M. Takano, Y. Takeda, R. Kanno and Y. Bando, Jpn. J. Appl. Phys., 27, L580-L583 (1988). 